\title{
Opportunistic Routing Protocol Based on Geographical Location and Energy Balance
}

\author{
Huirong Luo ${ }^{\text {a }}$, Li Wang ${ }^{\text {b, * }}$
}

School of Physics and Information Engineering, Jianghan University, Wuhan 430056, Hubei, China a hrluo1985@163.com, b, *9527cn@163.com

\begin{abstract}
In order to improve the life cycle of wireless sensor networks, An opportunistic routing protocol (GEBOR) based on geographic location and energy balance is proposed.The routing protocol comprehensively considers the transmission radius, link quality and residual energy threshold, and transmission energy efficiency (energy consumption/remaining energy), defines the new route metric. The node with the smallest route metric is selected as the next hop node.Finally, simulations are performed by OMNET++. The simulation results show that the proposed routing protocol can balance the energy consumption of nodes, prolong the lifetime of the nodes and the network, and improve the throughput of the system.
\end{abstract}

Keywords: transmission energy efficiency, link quality, residual energy, opportunity routing.

\section{Introduction}

With the rapid development of communication technology, the application of wireless sensor networks is more and more widely.As an important part of the development of wireless sensor network technology, opportunistic routing protocol has been a hot research topic. Most nodes in wireless sensor networks have limited energy and cannot be charged. Therefore, the focus of wireless sensor network routing protocol design is mainly on energy saving. In wireless sensor networks, the main task of a node is to collect data and transmit data. Transmiting data requires more energy than collecting data. Therefore, designing a path with the least energy consumption to transmit data is a priority in route designing. In addition, the remaining energy of the network node should be considered to prevent the node from dying prematurely, and the poor link quality and network topology cause the packet loss and the number of retransmissions to increase. For this, in 2005, the Extreme Opportunity Routing Protocol (ExOR) [1] was proposed.ExOR calculates the distance between the current node and the destination node based on the route measurement ETX(expected transmission count metric), taking into account factors such as the number of hops and the number of retransmissions, which greatly improves the forwarding rate of data packet. Michele et al. proposed the GeRaF[2] protocol, which selects forwarding nodes based on geographic location. Mao et al. proposed the Energy Efficient Opportunity Routing Protocol (EEOR) [3]. The EEOR protocol selects the node with the smallest energy consumption as the candidate forwarding node. Literature [4] proposes a Geographic Opportunity Routing Protocol (TLG) based on topology and link quality, which takes into account link quality, geographic location and residual energy as routing metrics. In addition, when the network is busy, a node with high priority needs to forward a large number of data packets, resulting in packet queuing, causing end-to-end delay and reducing network throughput.

In order to solve the problem, this paper proposes a new routing algorithm. Based on the geographical location strategy, considering the geographical location information, link quality, residual energy, transmission energy efficiency and node buffer, the opportunity routing protocol GEBOR based on geographic location and energy balance is proposed, and the performance of this protocol is simulated. 


\section{The GEBOR Protocol}

\subsection{Network Assumptions}

We assume the following:There are $\mathrm{n}$ nodes in the wireless sensor network randomly distributed in the rectangular of $\mathrm{M}^{*} \mathrm{M}$; all node positions are fixed, and each node can obtain its own position through GPS positioning; any node U, V must satisfy the distance of the two points $|U V|<R$ can be communicated, where $\mathrm{R}$ is the transmission radius.

\subsection{Routing Metric}

In an actual network environment, nodes with high residual energy or good link quality are selected for data forwarding. This will cause some nodes with good link quality or high residual energy to continuously forward data and die prematurely. In order to ensure the integrity of the network, the residual energy threshold and the link quality threshold are set. Below the threshold, the priority of the node is lowered, so that the network energy is evenly distributed. In addition, some nodes with high priority need to forward a large amount of data, which leads to queuing, resulting in end-to-end delay and reducing network throughput. In order to solve this problem, we can reduce the priority of nodes with too many packets.

The energy consumption when transmitting data is also an important consideration,so the transmission energy efficiency (energy consumption/remaining energy) is defined. Therefore, in the candidate forwarding set, we select multiple routing metrics to select the forwarding node of the next hop, including link quality, transmission energy efficiency, and node buff-ering,to improve the life cycle and throughput of the network. The proposed routing metric GEBOR is calculated according to (1).

$$
G E B O R=\alpha \times E T X+\beta \times \mathrm{EE}+\gamma \times \mathrm{Buf}
$$

ETX is the expected number of transmissions. The smaller the value is, the better the link quality is. EE is the transmission energy efficiency, the smaller the value, the smaller the relative energy consumption and the larger the remaining energy. Buf is the number of cached packets. The smaller the value, the smaller the number of packets waiting in the queue and the higher priority. $\alpha, \beta$ and $\gamma$ are the weights of ench metri and $\alpha+\beta+\gamma=1$.

\subsection{Link Quality}

Douglas et al. proposed the expected number of transmissions (ETX) [5] as a route metric to indicate the quality of the link. ETX refers to the number of expected transmissions that a node successfully sends a packet. The calculation of ETX consists of two parts, one is the number of forward forwarding $p_{f}$, and the other is the success rate of the response packet ACK $p_{r}$, so the expected probability of successfully transmitting a packet is $p_{f} * p_{r}$. The process of every attempt to send a packet can be considered a Bernoulli experiment, so the expected number of transmissions ETX is calculated according to (2).

$$
E T X=\frac{1}{p_{f} * p_{r}}
$$

The forwarding rate can be calculated by sending a link detection packet. The node sends a fixedsize probe packet every $\tau$ seconds. In order to prevent all nodes from transmitting at the same time causing synchronization effects, $0.1 \tau$ jitter transmission is performed between different nodes. In this way, each node only needs to record the number of probe packets received in the past w seconds, and then calculate the forwarding rate according to (3).

$$
p_{t}=\frac{\operatorname{count}(t-w, t)}{w / \tau}
$$

Where count $(t-w, t)$ refers to the number of probe packets actually received in $\mathrm{W}$ seconds; $w / \tau$ refers to the number of probe packets that should be received within $\mathrm{W}$ seconds. Therefore, according 
to formula (3), $p_{f}$ and $p_{r}$ can be calculated, and ETX (link quality) can be obtained according to formula (2).

\subsection{Transmission Energy Efficiency}

In order to extend the life cycle of the network, considering the impact of energy consumption and residual energy on the network, the transmission energy efficiency (EE)is defined, and the EE of node $i$ is defined as (4).

$$
E E_{i}=\frac{E_{i c}}{R E_{i}}
$$

Where $E_{i c}$ is the energy consumed by node $\mathrm{i}$ and $R E_{i}$ is the residual energy of node $\mathrm{i}$.

Selecting candidate sets through EE can avoid early death of some important nodes and cause network paralysis. For example, node s has two candidate nodes v1, v2, and assumes they have the same link quality. The remaining energy of $\mathrm{v} 1$ and $\mathrm{v} 2$ is 1 unit and 8 units respectively. Forwarding a data packet to the destination node requires 2 units and 4 units of energy respectively. According to Equation 4, the EE is 2 and 0.5 respectively, then the node v2 with small EE is selected as the forwarding node, instead of the $\mathrm{v} 1$ with low energy consumption as the forwarding node. Therefore, compared with EEOR opportunistic routing, this method can effectively improve the network life cycle.

\subsubsection{Node Energy Consumption Model}

Considering the limited energy of the node battery in the wireless sensor network and the inconvenience of replacing the battery, energy consumption is an important consideration in routing metrics. In this paper, the energy consumption model [6] is used to describe the energy required to send and receive a packet. The transmission power of the node i successfully transmitting k-bit data packet to the node $\mathrm{j}$ is:

$$
E_{T x}=\left\{\begin{array}{l}
k\left(E_{\text {elec }}+E_{f s} d_{i, j}^{2}\right), \quad d_{i, j}<d_{0} \\
k\left(E_{\text {elec }}+E_{a m p} d_{i, j}^{4}\right), \quad d_{i, j} \geq d_{0}
\end{array}\right.
$$

Where $E_{\text {elec }}$ is the energy consumed to forward or receive one bit of data, $E_{f s}$ and $E_{a m p}$ are the circuit amplification factors of the free space model and the multipath attenuation model, respectively, and the amplification factor is constant. $d_{0}$ is the transmission distance threshold.

The energy consumed by node $\mathrm{j}$ to accept k-bit packets is calculated by (6).

$$
E_{R x}=k \times E_{\text {elec }}
$$

\subsubsection{Energy Consumption}

The energy consumption[7] of node i successfully transmitting a packet to the next hop is recorded as $E_{i C}$, and it consists of three parts: $E_{i T}$ is the energy consumed to send a packet, $E_{i R}$ is the energy consumed to receive a packet, and $E_{i A c k}$ is the energy consumed to send an ACK response packet. The total energy consumption $E_{i c}$ is calculated by Equation (7).

$$
E_{i C}=E_{i T}+N_{c} \times E_{i R}+E_{i A c k}
$$

Where $N_{c}$ is the number of neighbor nodes of node $\mathrm{i}$ within communication distance $\mathrm{R}$.

Therefore, the residual energy of node i can be expressed by Equation (8).

$$
R E_{i}=E_{0}-E_{i C}=\sum_{j=1}^{m} \sum_{k=1}^{n} \frac{w_{j}^{k}}{p k t s i z e}\left(E_{i T}+N_{c} \times E_{i R}+E_{i A c k}\right)
$$

Where $E_{0}$ is the initial energy of node $\mathrm{i}, \mathrm{n}$ is the number of streams for node $\mathrm{i}, \mathrm{m}$ is the number of types of streams, $w_{j}^{k}$ is the total number of bits of $\mathrm{k}$ data streams in class $\mathrm{j}$, and pktsize is the size of the packet. 


\section{Routing Protocol Design}

\subsection{Routing Strategy Analysis}

As shown in Fig. 1, Src is the source node, Dst is the destination node, and A, B, C, D, E, and F in $\mathrm{C} 2$ are neighbor nodes of the source node Src. The source node Src exchanges information with the neighbor nodes, obtains the ID number and location information of the neighbor nodes, and calculates the distance between the two nodes. If the distance is greater than the transmission radius $\mathrm{R}$, the node is discarded, otherwise the node is added to the competition candidate node set. The node that satisfies the link quality and the residual energy constraint is selected as the candidate forwarding node set in this contention candidate node set, and is denoted as $\mathrm{C} 1$. The route metric between the source node and the candidate forwarding node is calculated according to formula (1). The smaller the route metric is, the higher the priority is, and the node with the highest priority is selected as the next hop forwarding node.

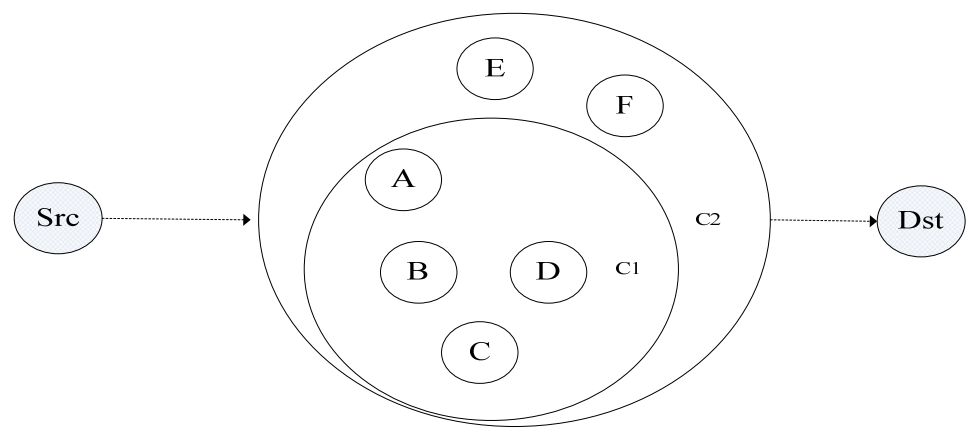

Fig. 1 candidate nodes and neighbor nodes

\subsection{Route Establishment Process}

First, a link model for threshold setting of transmission radius, link quality and residual energy is proposed. Let the transmission radius threshold be $\mathrm{R}$, the link quality threshold be $Q_{0}$, and the residual energy threshold be $E_{0}$. The link quality threshold is the probability of successfully transmitting a data packet. The remaining energy threshold $E_{0}$ is the minimum energy required to successfully send a packet and send a response packet to the previous node. This avoids the energy of a node being too small to be successfully transmitted, and the premature death of the node causing the network to crash. Only the node that satisfies the distance between the nodes is smaller than the transmission distance threshold $\mathrm{R}$, and the distance from the neighbor node to the destination node is smaller than the distance from the source node to the destination node can join the competition candidate set $\mathrm{S}$. The node whose link quality and residual energy is greater than the threshold in $\mathrm{S}$ joins the candidate forwarding node.

As shown in Fig. 2, m neighbor nodes are distributed around the node n, and the ID number, location information, and link quality and remaining energy of the neighbor node are known by sending a request and reply. The specific process of route establishment is as follows: .

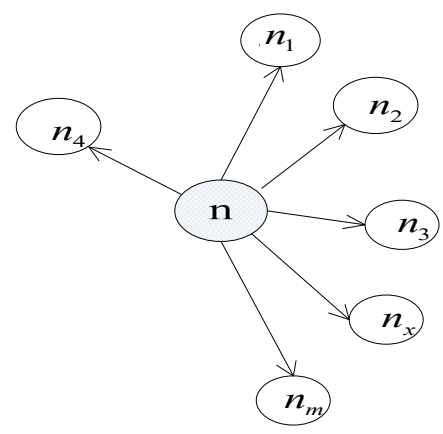

Fig.2 Route establishment for nodes 
(1) Node $\mathrm{n}$ sends request information and collects information from neighbor nodes. Node_ID denotes the ID number of node $\mathrm{n}$, REQ denotes the request information, $\left(x_{n}, y_{n}\right)$ is the coordinate of node $n$.

Table 1. Source node information

\begin{tabular}{l} 
Node_ID \\
\hline (2) After receiving the request information, any neighbor node $\mathrm{x}$ collects its own location \\
information, remaining energy information, link quality, and number of nodes buffered data packets, \\
and replies with a response signal ACK, as shown in the table2. Where Node_IDs is the ID number \\
of the $\mathrm{x}$ node, Node_IDd is the destination node ID number, $\left(x_{x}, y_{x}\right)$ is the coordinate of $\mathrm{x}$, and $E_{r e s}$ \\
and $Q_{x}$ are the remaining energy and link quality information of $\mathrm{x}$, respectively. Buf indicates the \\
number of buffered packets.
\end{tabular}

Table 2. Neighbor node information

$\begin{array}{llllllll}\text { Node_IDs } & \text { Node_IDd } & \text { ACK } & \mathrm{x}_{\mathrm{x}} & \mathrm{y}_{\mathrm{x}} & \mathrm{E}_{\mathrm{res}} & \mathrm{Q}_{\mathrm{x}} & \mathrm{Buf}\end{array}$

(3) The node $\mathrm{n}$ calculates the distance $d_{n x}$ between the node $\mathrm{n}$ and the $\mathrm{x}$ according to the received response information. If $\left|d_{n x}\right| \leq R$, and the distance from the neighbor node $\mathbf{x}$ to the destination node Dst is smaller than the distance from the source node $\mathrm{n}$ to the destination node, Then the node is added to the competition forwarding candidates set $U$, otherwise the node is discarded.

(4) In the competition forwarding candidates set $\mathrm{U}$, if the node $\mathrm{x}$ satisfies $Q_{x} \geq Q_{0}$ and $E_{r e s} \geq E_{0}$, the node joins the forwarding candidates set $\mathrm{V}$.

(5) The routing metrics of the nodes are calculated according to formula (1) in the forwarding candidate set $\mathrm{V}$, and the routing metrics are prioritized from small to large. The smaller the routing metrics, the higher the priority, and the highest priority is the forwarding node.

\section{Simulation and Analysis}

The simulation experiment was completed using the OMNET ++4 simulation platform, and the improved opportunity routing protocol GEBOR was compared with the classic ExOR protocol. We set the simulation parameters as follows: the wireless sensor nodes are randomly distributed in the area of $200 * 200 \mathrm{~m}$; the number of nodes is 200 ; the transmission radius is $30 \mathrm{~m}$; the packet size is 150 bytes; the initial energy of the node is $1 \mathrm{~J}$; the threshold of the link quality is 0.4 , and the threshold of the remaining energy is $0.002 \mathrm{~J}$.

\subsection{Network Lifetime}

Fig. 3 shows the relationship between network lifetime and transmission radius. It can be seen that as the transmission radius increases, the network lifetime becomes smaller. Because if the transmission radius is larger, the required transmission power is also larger, resulting in less lifetime of the entire network. In the two opportunistic routes, GEBOR has a longer network lifetime. Because the selection of the candidate set not only considers the threshold of link quality and the residual energy, but also considers the transmission energy efficiency, which balances the energy consumption of the entire network, thereby prolonging the network lifetime. 


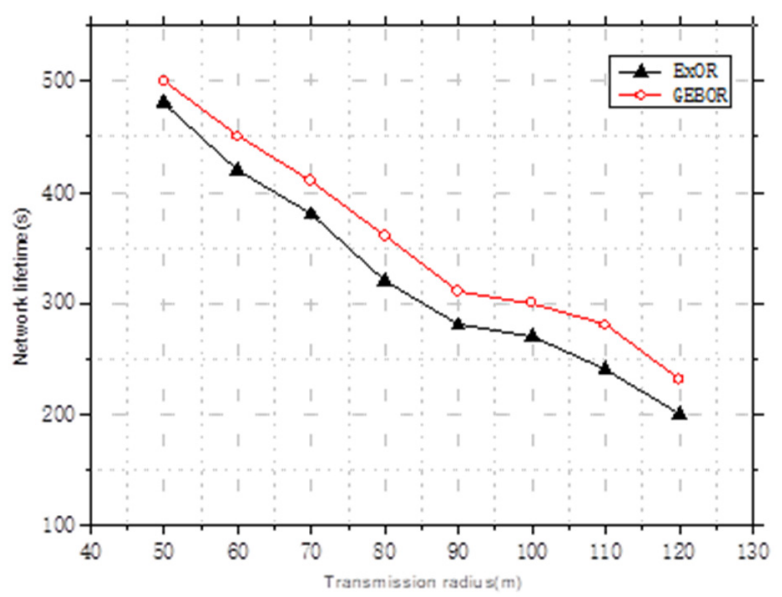

Fig. 3 Relationship between network lifetime and transmission radius

\subsection{Number of Network Nodes}

Fig. 4 shows the comparison between the GEBOR and the classic ExOR protocol on the number of network nodes surviving. As can be seen from Fig. 4, as the transmission time increases, the node gradually dies. In the first 20 seconds, the node deaths of both protocols are 0 . After that, the node of the classic ExOR protocol begins to die, while the GEBOR protocol begins to die after 40 seconds. The life cycle of the network is defined as the time at which the first node dies . Therefore, compared to EXOR, the GEBOR protocol can extend the network lifetime.

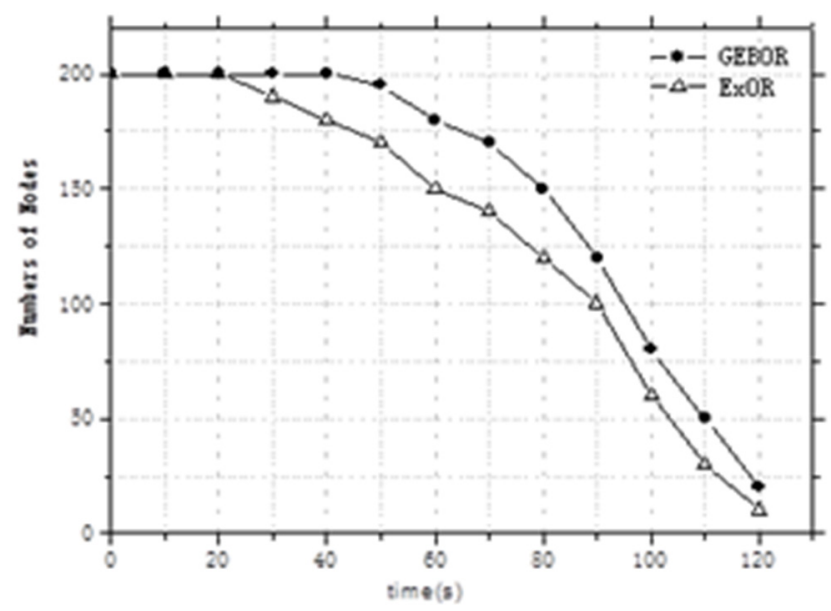

Fig. 4 Number of nodes

\subsection{Network Throughput}

Network throughput is an important indicator to measure the performance of the network. As can be seen from Fig. 5, the throughput of the two protocols is basically the same before 30 seconds, and at 30 seconds, the node of the ExOR protocol starts to die, so the throughput of the EXOR protocol decreases. In the GEBOR protocol of this paper, in the selection of candidate forwarding nodes, not only the transmission distance is considered, but also the link quality threshold and the residual energy threshold are set, combined with the transmission energy efficiency, the energy of the network is balanced, and the throughput of the network is improved. 


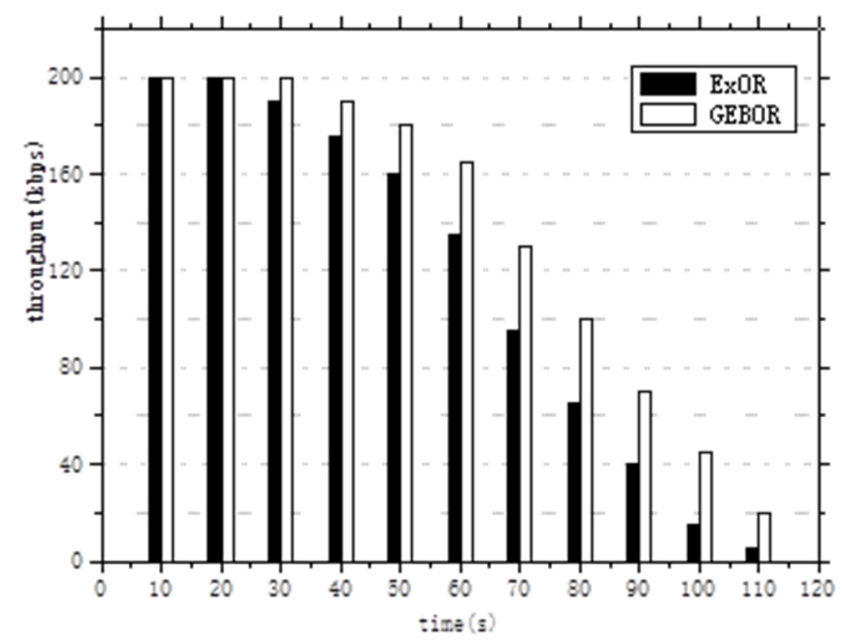

Fig. 5 Throughput of the two protocols

\section{Conclusion}

This paper proposes an opportunity routing protocol GEBOR based on geographic location and energy balance for the problem of premature death of nodes in wireless sensor networks. The protocol comprehensively considers the geographic location, link quality, threshold of residual energy, and transmission energy efficiency of nodes in the selection of candidate forwarding sets, thereby extending the life cycle of the network. The experimental results show that the GEBOR protocol is superior to the classic ExOR protocol in extending the life cycle of the network and improving the throughput of the network.

\section{References}

[1]. Biswas S, Morris R. ExOR: Opportunistic Mult-i hop Routing for Wireless Networks [C]. Proceedings of the 2005 Conference on Applications, Technologies, Architecture, and Protocols for Computer Communications.New York:ACM,2005:133-144.

[2]. Michele Z, Ramesh RR. Geographic random forwarding (GeRaF) for ad hoc and sensor.

[3]. networks: energy and latency performance[J]. IEEE Transactions on Mobile Computing, 2003, 2(4):349-365.

[4]. Mao Xufei, Xu Xiaohua, Tang Shaojie, et al. Energy-efficient opportunistic routing in wirele-ss sensor networks[J]. IEEE Transactions on Parallel and Distributed Systems,2011, 22(11): 19341942.

[5]. Zhao Z, Rosario D, Braun T, et al. Topology and link qualityaware geographical opportuni-stic routing in wireless ad-hoc networks[C]. International wireless communications and mobile computing conference,2013: 1522-1527.

[6]. Douglas S. J, De Couto, Daniel Aguayo, et al. A high-throughput path metric for multi-hop wireless routing[J]. Wireless Networks,2003,11(4): 419-434.

[7]. Yisheng Miao, Huari Wu, and Huaji Zhu. Research on Power Consumption Weighted State Machine Base on Cluster Head Cycle-Switching WSN node [J]. Computer Engineering and Design, 2014(8): 2723-2727.

[8]. Yang Qin, Li Li, Xiaoxiong Zhong, etc. Opportunistic routing with admission control in wireless ad hoc networks[J]. Computer Communications 2015(55):32-40. 